\title{
CLINICAL REFLECTION \\ Parity of esteem: evaluating inequalities in older adult healthcare
}

\author{
James Ashcroft \& Gareth Thomas
}

\begin{abstract}
Correspondence Dr James Ashcroft, Imperial College London, South Kensington Campus, London SW7 2AZ, UK. Email: james.ashcroft17@imperial.ac.uk
\end{abstract}

\section{Copyright and usage}

(C) The Royal College of Psychiatrists 2018

\section{SUMMARY}

Addressing inequalities between mental and physical healthcare in older adult healthcare is imperative for safe patient care. This evaluation of services at The Harbour mental health hospital, Blackpool, UK gives insight into parity of esteem and prompts investigation into the clinical decisions of doctors working in older adult mental healthcare.

\section{DECLARATION OF INTEREST}

None.

In a previous issue of BJPsych Advances, Mitchell, Hardy \& Shiers published an in-depth evaluation and provoking article concerning parity of esteem in mental and physical healthcare (Mitchell 2017). It is of utmost importance not only to understand the concept of parity of esteem, but also to be aware of its damaging effects and make strategies to combat these in order to provide safe patient care.

\section{A study of admission procedures}

To investigate parity of esteem at The Harbour mental health hospital, Blackpool, UK (part of Lancashire Care NHS Foundation Trust), standard operations procedure (SOP) parameters recorded on admission were reviewed for 64 patients admitted to the older adult ward throughout March 2017. The SOP includes both mental health parameters (psychiatric history, mental state examination, informant history, risk assessment, drug chart assessment) and physical health parameters (medical history, systems review, physical examination, physical observations, blood tests, electrocardiogram, midstream specimen of urine analysis). The most frequently assessed parameters were mental state examination and psychiatric history, with physical health parameters being assessed notably more infrequently than mental health parameters. These findings were presented at a local quality improvement meeting, SOP guidance was made visible on the wards, and educational sessions were implemented on the patient admission process. Following this implementation, the SOP parameters of the 38 patients admitted to the older adult ward throughout July were reviewed. This review revealed that the frequency of assessment of the majority of physical and mental health parameters remained largely unchanged ( $\leq 10 \%$ difference).

As a result of this brief study, an expanded induction session, an out-of-hours clerking process session and a clerking rationale educational session have been introduced to emphasise the importance of complete health parameter assessment on admission. Further changes are being discussed by The Harbour's Trust Medical Education Committee regarding subjects such as: accessibility of equipment, paper and computer systems used by the trust, the role of the multidisciplinary team in the initial assessment of in-patients, and methods to gain further insight into parity of esteem.

\section{Discussion}

\section{Limitations of our study}

Although reviewing SOP parameters sheds some light on parity of esteem in mental healthcare it is not a comprehensive investigation. The SOP offers only a snapshot of the complete evaluation of patients, making it difficult to establish whether disparity in the assessment process is directly linked to clinical decision-making. However, The Harbour's SOP provides an easily accessible set of measurements and gives an indication of attitudes towards the importance of mental and physical healthcare in an older adult mental health setting. The older adult demographic is a complex patient group with frailty, multiple comorbidity and polypharmacy requiring thorough evaluation and assessment. Standards for older adult in-patient mental health services published by the Royal College of Psychiatrists (Bolger 2017) recommend that a number of physical health assessments (such as physical observations and medication review) are performed, but many of the physical assessments (such as a systems review and lifestyle factors) are to be completed within 1 week of admission and are therefore outside of the scope of the SOP, which records parameters solely on admission. This indicates that our snapshot may not be the most realistic approach to assessing inequalities between mental and physical healthcare. Furthermore, other considerations must be taken 
into account in our evaluation of the SOP which may have affected the assessment of patients, such as: time constraints, staff availability, access to equipment and patient variability.

\section{Suggestions for the immediate future}

This evaluation does prompt further investigation into the clinical decisions of doctors working in older adult mental healthcare. The British Medical Association's parity of esteem recommendations highlighted by Mitchell and colleagues (Mitchell 2017) are positive steps forwards in the junior doctor training pathway. We have struggled to find an effective solution to the inequality in admission clerking present at The Harbour. Increased training and education did not influence trainee decision-making concerning parity of esteem and we believe that the thoughts and decision-making of trainees in mental healthcare is an area underrepresented in current psychiatric research and underappreciated in current recommendations. Increased exposure to psychiatry through integrated mental health trainee rotations and mental health awareness training will influence decision-making through exposure. However, at present we suggest that more investigations are undertaken with foundation year doctors, core medical trainees and young psychiatry specialty trainees to aid in identifying potential causes for the reduced frequency of physical examinations during psychiatric admissions found in our study and current views on parity of esteem. This research could benefit from a qualitative approach which could guide future education of trainees and which should not only emphasise the importance of physical assessments, but also attempt to change perceptions of the assessments required when admitting psychiatric patients.

Trainee physicians are the new generation of psychiatrists and are incredibly important to the specialty. Mitchell and colleague's article and the results of our closed-loop quality improvement study necessitate exploration of the reasons behind the views and actions of trainees that goes beyond increased exposure to the specialty.

\section{References}

Bolger H, Parker E (2017) Standards for Inpatient Older Adults Mental Health Services: Fourth Edition (CCOI257). College Centre for Quality Improvement, Royal College of Psychiatrists.

Mitchell AJ, Hardy S, Shiers D (2017) Parity of esteem: addressing the inequalities between mental and physical healthcare. BJPsych Advances, 23: 196-205. 